\title{
Software Engineering Based Fault Tolerance Method for Wireless Sensor Network
}

\author{
Sama Hussam Sabah Sabah ${ }^{1}$, Muayad Sadik Croock ${ }^{2}$ \\ ${ }^{1,2}$ Computer Engineering Department, University of Technology-Iraq, Baghdad, Iraq \\ 120634@student.uotechnology.edu.iq, Muayad.S.Croock@uotechnology.edu.iq
}

\begin{abstract}
The management of faults in Wireless Sensor Networks (WSN) has been considered recently. The problem of tolerating the detected fault is solved by presenting different methods from numerous researchers. Moreover, the software engineering approaches have been adopted to introduce methods with high reliability. In this paper, a fault tolerance method is proposed for WSN based on the software engineering self-checking process to deal with the faults that affect energy consumption in the network and make it drop earlier. The proposed method detects the appeared fault at any sensor node and recovers the faulted readings by computing the average value of its neighbor nodes. In addition, this process is continued until the faulty sensor is fixed by the maintenance team. The proposed method is tested over different case studies and the obtained results prove the claim of the paper's idea.
\end{abstract}

Index Terms - WSN, Software Engineering, Fault Tolerance, Self-Checking Process.

\section{INTRODUCTION}

Wireless Sensor Network (WSN) contains a huge number of tiny sensors that are distributed formally or informally over an area to be monitored. These sensors are the basic unit of such network because it does the process of observing (sensing) the physical parameters (like temperature, pressure, humidity,.., etc) of the environment in the location where it is placed and sent these values through a proper path [1]-[2].

These individual sensors are liable to un expected failure, such as environmental interference, physical damage, load data to forward, ... etc [3]-[4]. This leads to send incorrect data and some faults make these sensors to consume more energy and die early or even the congestion that leads to spend a huge energy in sending data. These faults are divided into physical and software [5]. In this paper, there is a focus on these types of errors to reduce the consumed power under the limitation of energy of battery [6]. Therefore, a proposed algorithm for controlling these faults and creating a recovery solution as a part of fault tolerance. It is very important to note that the WSN needs to exhibit a low probability of false and high probability of fault identification. A faulty sensor node means that there is incorrect information that affects the performance of any task. Therefore, a recovering method is required to increase the reliability [7]-[8]. The wireless sensor networks can be dynamically adapted to any sensor failures and reacted to changes in the network environment [9]. It is also measured to recognize, adapt, and anticipate the power consumption of sensors [10]. As mentioned earlier, the controlling of power consumption is adjusted using fault tolerance technique as the faults are detected depending on the consumed power of each sensor node.

In this paper, a fault tolerance method, based on the software engineering process, is proposed. The fault tolerance method consists of two steps. The first step is the detection of 
the faulted sensors using the self-checking process that uses threshold values to control the consumed power. The second step is the recovery of the detected fault by computing the average value of neighbor nodes to keep the continuity of network working. This operation is continued till the fault is fixed by maintenance team.

\section{RELATED WORKS}

In this section a number of algorithms and protocols have been reviewed for the purpose of using software engineering techniques and managing power consumption in WSN. In [3], the authors customized an approach to optimize the energy consumption in WSN. It has used the K-means clustering algorithm to find the shortest path with low cost data transfer and less error rate from sensor node to sink node and optimize this path by using backpropagation algorithm to remove unwanted data loss and data transfer. This technique aimed to manage the energy consumption by overriding the occurrence of faults and prolong life time of WSN. In [5] new energy efficient based fault tolerance protocol for WSN [EFP protocol] was proposed that aimed to decrease the consumption of the network resources like energy. This EFP protocol made improvement on leach algorithm that selected cluster head based on the receiving signal strength. All nodes have the same initial energy, then creating TDMA Schedule and assigning each node a time slot to transmit means that they distributed is impartially in leach algorithm

In [6], fault tolerance and energy efficient clustering algorithm in WSN was suggested to improve clustering-based energy efficiency and fault tolerance. This algorithm was used to cluster nodes and selected cluster head $(\mathrm{CH})$. Each $\mathrm{CH}$ has a backup node called back up cluster head $(\mathrm{BCH})$ to supervise the performance of $\mathrm{CH}$ and saving copy of the data of $\mathrm{CH}$, when fault happens in $\mathrm{CH}$ direct change to $\mathrm{BCH}$ until fault is fixed.

In addition, after collecting data from member's nodes, it detected and isolated the faulty node using weighted medium. The faulty node was replaced by a neighboring node that changes its status from sleep mode to wake up mode. The results showed a significant improvement in energy and data management. Also in ref [11] a new protocol was proposed to enhance the performance of network in terms of finding out the shortest energy density route so that for communicating small amount of consumed power and that increases the life time of the network by overcoming the faults that cause an increase in energy .

\section{PROPOSED SYSTEM}

As mentioned earlier, the proposed method tackles the problem of detecting a fault inside the WSN. This is done by using software engineering approach to design the proposed method. The next three subsections discuss the proposed system to make the reading flow easier.

\section{A. SYSTEM STRUCTURE}

In this section, the structure of the proposed method for WSN is shown in Figure (1). It is important to note that all nodes of the considered WSN are homogeneous with the same characteristics. 


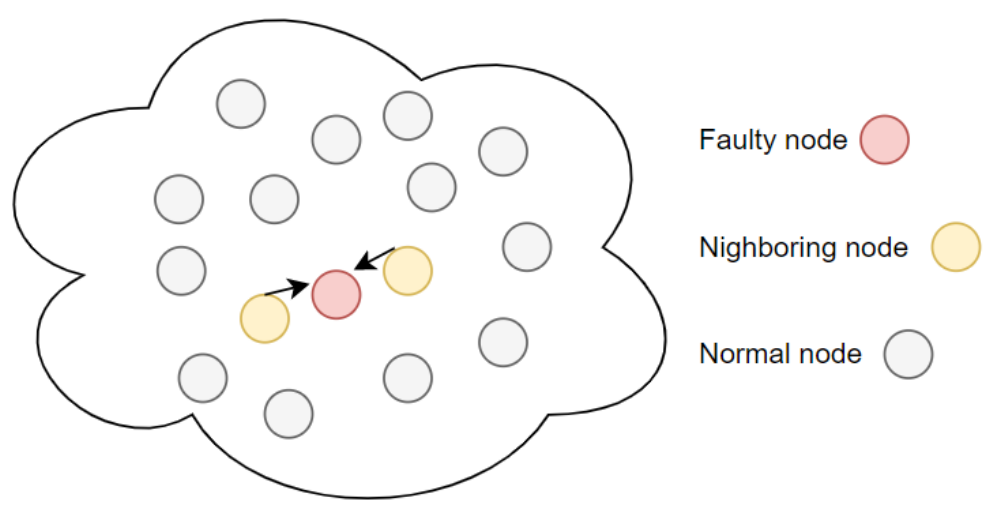

FIG. 1. THE STRUCTURE OF THE SYSTEM.

The considered WSN contains a number of sensor nodes, which are distributed in network randomly. Each node can communicate with other nodes in the WSN by using AODV routing protocol to simulate a real network that consists of source and destination and paths, ..., etc

Throughout the working of the network, each sensor node consumes power for transmitting the data and other communication signals between nodes . Meanwhile, each node can suffer from faults in both types of software and hardware. Both faults can consume power more than the normal as a reason for over transmission loading and physical faults.

\section{B. PROPOSED FAULT TOLERANCE ALGORITHM}

The proposed fault tolerance algorithm represents the backbone of the method that manages the detection and recovery of the faulted sensors. The fault tolerance uses the selfchecking process as a method of fault detection. This is done by allocating an upper and lower threshold values for considering the normal power consumption and then continued checking for measuring power consumption in each node for detecting the fault efficiently. In this section the details of the proposed algorithm are shown in Figure (2) as a flowchart. The adopted steps of the algorithm are explained as:

Step 1: each node computes the quantity of power consumption and storage.

Step 2: each node is checked frequently using the self-checking for any possible fault.

Step 3: If there is a fault detection, the node is allocated and changed to sleep mode.

Step 4: The readings from neighbor nodes of the faulted nodes are collected by sending navigation to take their readings.

Step 5: The proposed algorithm estimates the value of faulted node by averaging the neighbor values and send it to the sink.

Step 6: The proposed algorithm checks the residual energy ratio of the sensor. If it is more than $50 \%$, the sensor goes back to normal state. Otherwise, the fault is occurred and the maintenance team is informed, also it turns to sleep mode.

Step 7: If the residual energy of sensor is less than 50\%, it remains at sleep mode and when reading is compensated from two nearest neighbors. 


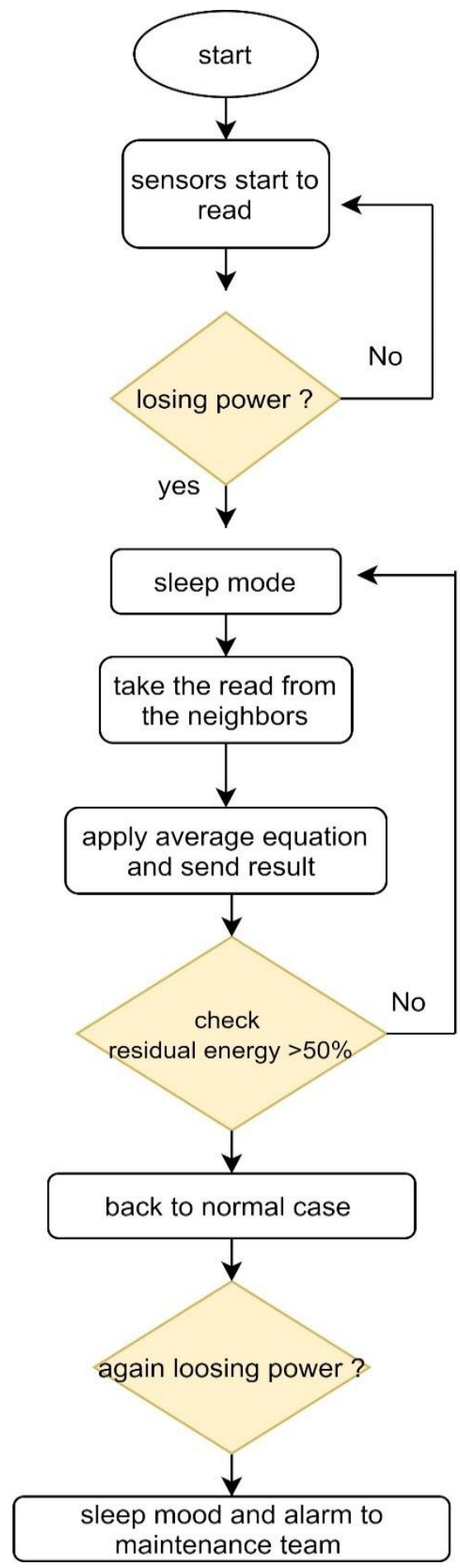

FIG. 2. FLOWCHART OF THE PROPOSED ALGORITHM 


\section{SIMULATION RESULTS}

In order to test the proposed algorithm, a fault is injected in an allocated node to show that it is faulted. It is detected by using self-checking process, the node is immediately recovered by using the proposed algorithm, as explained above.

In this section, the OMNET++ simulator is adopted to build and apply the proposed algorithm to realize the real-time working of the sensor network and it is adopted network simulator. Figure (3) shows the adopted WSN that distributes 20 sensor nodes as a prototype that can clarify the idea of the proposed algorithm. Each node pings to other nodes to record the distances between them. While, the AODV protocol is considered for as a stander protocol that manages the routing and communication processes between the nodes. The network is started normally, and all nodes send request to transmit and receive acknowledge from other nodes and then working to give us some of the reading of the network.

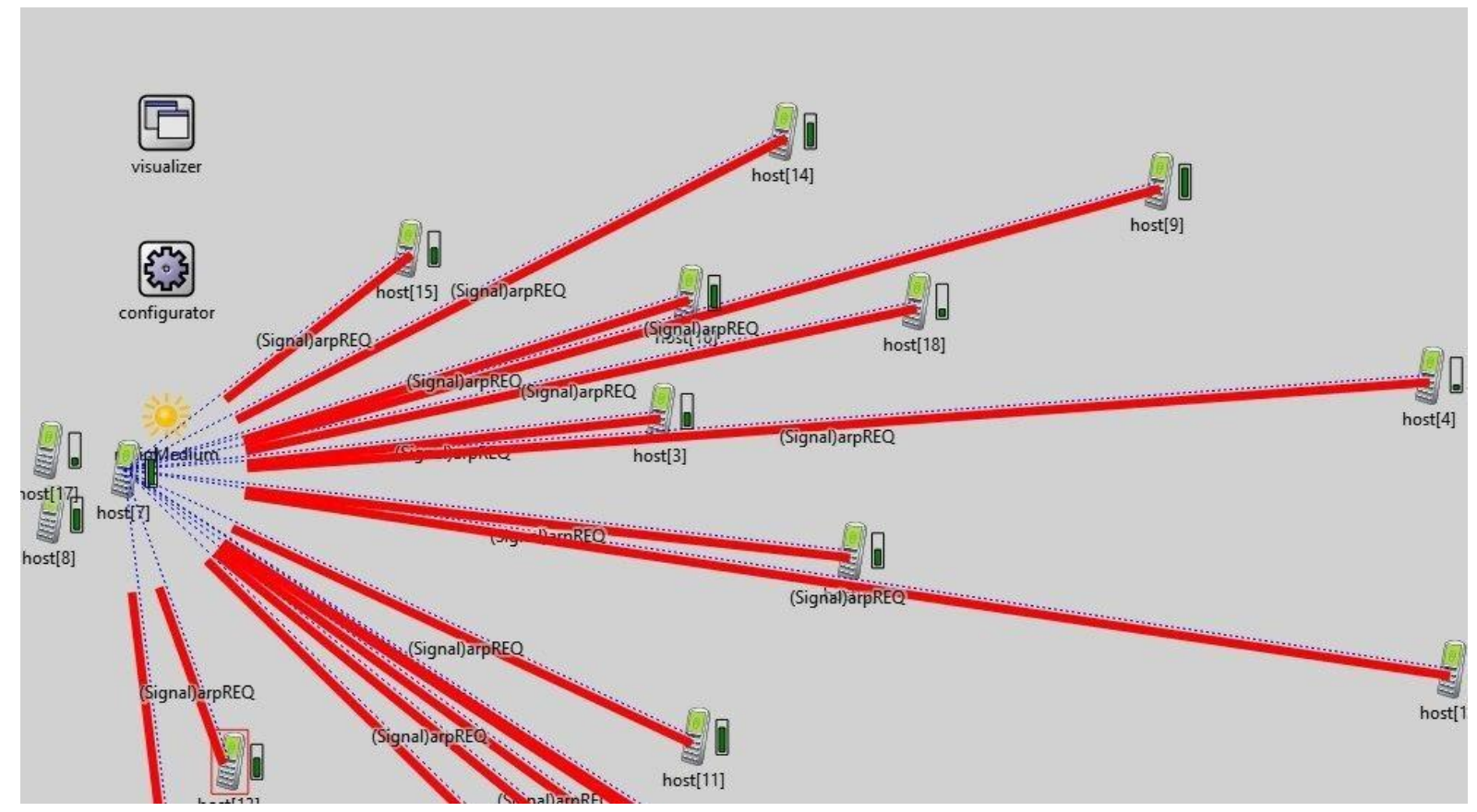

FIG. 3. THE BUILDING OF THE NETWORK.

In Figure (3), the red lines represent the links between sensors for guaranteeing the communications between them. Each sensor owns energy storage module that contains an energy balance watched variable. This watch can be used to track energy consumption to detect faults when consuming power become more than threshold values. Each telephone icon means sensor module. While the sun icon means the radio medium module as a configurator module assigned IP addresses to the hosts and visualization module is to show radio signals.

Figure (4) shows the detection of the faulted node by using the proposed algorithm, explained earlier, that is based on the consumed power by each node individually. In the simulation and as a case study for multiple sensor nodes, the faults are injected inside the network to node host [5], host [12], and host [15] that are allocated in the red cross signal. The proposed method firstly detects the nodes that crosses the allowed threshold levels of consumed power, which are hosts of [5], [12], and [15]. The reading values of these nodes are zeros, before applying the second part of the proposed algorithm, as they are set to sleep mode as shown in Table (1). The proposed method secondly recovers the faulted nodes by using the neighbor nodes as a tolerant way for compensating the zero values by average 
value of neighbors, as shown in Table (2). Neighbor nodes are selected depending on the distance between them and faulty node (short distance). From the Figure and Tables, it is proved that the proposed algorithm performs the recovery and fault tolerance concept in a proper way.

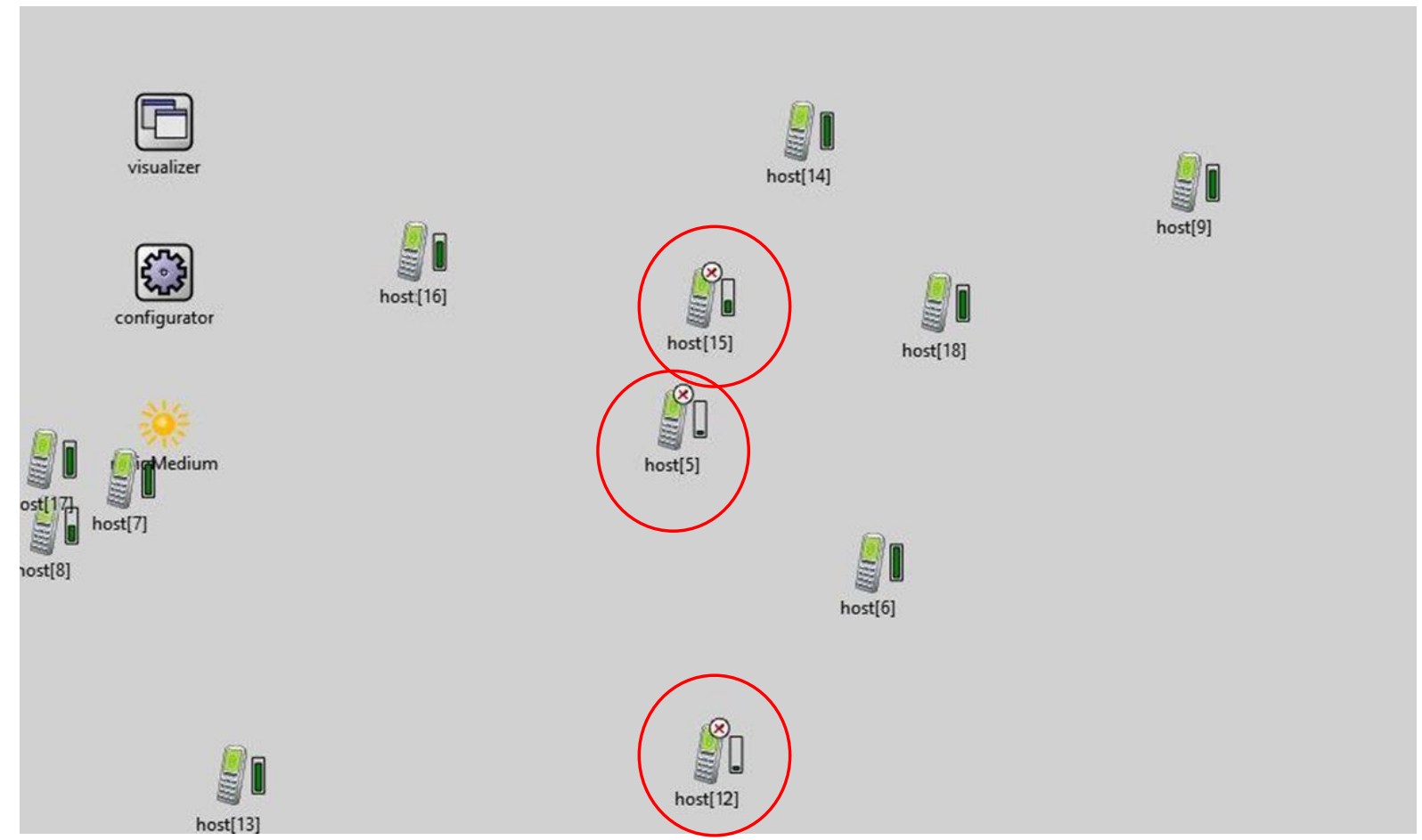

Fig.4. DETECTION OF FAULTY NODE.

TABLE 1. SENSOR NODE READING BEFORE APPLYING THE PROPOSED ALGORITHM

\begin{tabular}{|c|c|c|}
\hline No. & Node host & Sensor readings \\
\hline 1 & Host [0] & 470 \\
\hline 2 & Host [1] & 470 \\
\hline 3 & Host [2] & 470 \\
\hline 4 & Host [3] & 471 \\
\hline 5 & Host [4] & 471 \\
\hline 6 & Host [5] & $\mathbf{0}$ \\
\hline 7 & Host [6] & 470 \\
\hline 8 & Host [7] & 470 \\
\hline 9 & Host [8] & 471 \\
\hline 10 & Host [9] & 470 \\
\hline 11 & Host [10] & 471 \\
\hline 12 & Host [11] & 471 \\
\hline 13 & Host [12] & $\mathbf{0}$ \\
\hline 14 & Host [13] & 470 \\
\hline 15 & Host [14] & 472 \\
\hline 16 & Host [15] & $\mathbf{0}$ \\
\hline 17 & Host [16] & 471 \\
\hline 18 & Host [17] & 471 \\
\hline 19 & Host [18] & 471 \\
\hline
\end{tabular}


Table 2. SENSOR NODE READING AFTER APPLYING THE PROPOSED ALGORITHM

\begin{tabular}{ccc}
\hline No. & Node host & Sensor readings \\
\hline 1 & Host [0] & 470 \\
2 & Host [1] & 470 \\
3 & Host [2] & 470 \\
4 & Host [3] & 471 \\
5 & Host [4] & 471 \\
6 & Host [5] & 470 \\
7 & Host [6] & 470 \\
8 & Host [7] & 470 \\
9 & Host [8] & 471 \\
10 & Host [9] & 470 \\
11 & Host [10] & 471 \\
12 & Host [11] & 471 \\
13 & Host [12] & 471 \\
14 & Host [13] & 470 \\
15 & Host [14] & 472 \\
16 & Host [15] & 471 \\
17 & Host [16] & Host [17] \\
19 & Host [18] & 471 \\
\hline & & 471 \\
\hline
\end{tabular}

It is important to note that the proposed method is compared with the research work of [3], [10], and [11]. The researchers have detected the faults in the sensor network in different way and found the solution by turning off these nodes. In this paper, the fault is detected based on self-checking process that is supported by software engineering techniques. This can increase the reliability and fault detection in terms of efficiency.

\section{CONCLUSION}

A Fault tolerance algorithm for WSN was proposed. The proposed algorithm used the software engineering self-checking process to detect the faulted nodes inside the WSN. This algorithm consisted of two sides: detection and recovery. The fault detection was performed using self-checking process. The faulty node was recovered by computing the value from neighbor nodes as a tolerant way till such node was fixed. It was important to note that the proposed method used fault tolerance technique to avoid high consuming of power in each node, individually, happened due to congestion or physical fault. The proposed algorithm was tested over a case study with multiple faulty nodes to test the behavior of it inside the network. The obtained results showed the efficient performance of it in terms of detecting the faults and recovering that proved the aim of the proposed algorithm. The proposed method might suffer from overlapping in a huge number of sensor node in a single network. Thus, the clustering method can be adopted in the future work to reduce the efforts of managing nodes in terms of power consumption and fault tolerance. 


\section{REFERENCES}

[1] Ashish,Gupta and Bhupender,Singh and Binary Kumar , “ Power Management in Wireless Sensor Networks “, International Journal of Advanced Research in Computer Science and Software Engineering,Vol.4,p.p.163-167,April 2014.

[2] ] Hitesh Mohapatra and Amiya.Kumar Rath " "Fault tolerance in WSN through PE-LEACH protocol ", The Institution of Engineering and Technology , Vol.9 , pp.358-365,2019

[3] ] Krishnapriya and Santosh,Anandh and Somnath,Sinha, " A Customised Approach for Reducing Energy Consumption in Wireless Sensor Network" , International Journal of Innovative Technology and Exploring Engineering (IJITEE), Vol.8,June 2019.

[4] Julia.Sosedka and Igor Shostko , "Calculation method for power consumption and lifetime of nodes in WSN", IEEE , October ,2014.

[5] Zohre Arabi and Roghayeh Parikhani , "EFP:NEW ENERGY-EFFICIENT FAULT-TOLERANT PROTOCOL FOR WIRELESSSENSOR NETWORK “ , International Journal of Computer Networks \& Communications (IJCNC) ,Vol.4, No.6, 2012.

[6] Elham Moridi and Somaya Jafarali, "Fault Tolerance and Energy Efficient Clustering Algorithm in Wireless Sensor Networks: FTEC “, Wireless Personal Communications, April 2019.

[7] Gholamreza Kakamanshadi and Savita Gupta and Sukhwinder Singh ," A Survey on Fault Tolerance Techniques in Wireless Sensor Networks ", InternationalConference on Green Computing and Internet of Things (ICGCIoT), 2015

[8] Ashutosh Choudhary ,'Review On Falut Tolerance in Wireless Sensor Network", international journal of computer application, vol.182, no.45, march 2019.

[9] K. Arulmozhi and V. R. Sarma Dhulipala and R. Prabakaran, "A study on optimized power consumption routing in Wireless Sensor Networks.”, IEEE, 2011.

[10] Doo-Soon " Fault Tolerance and Energy Consumption Scheme of a Wireless Sensor Network “ , International Journal of Distributed Sensor Networks , November 2013

[11] Manish,Bhardwaj and Anil Ahlawat and Nidhi Bansal ," Maximization of Lifetime of Wireless Sensor Network with Sensitive Power Dynamic Protocol “ , International Journal of Engineering \& Technology , 2018. 